\title{
Penser la langue, décrire le monde. Conversation avec Pierre Jourde
}

\section{Veronic Algeri}

\section{(2) OpenEdition}

Journals

\section{Édition électronique}

URL : http://journals.openedition.org/rief/1074

DOI : 10.4000/rief.1074

ISSN : 2240-7456

\section{Éditeur}

Seminario di filologia francese

\section{Référence électronique}

Veronic Algeri, «Penser la langue, décrire le monde. Conversation avec Pierre Jourde », Revue italienne d'études françaises [En ligne], 5 | 2015, mis en ligne le 15 décembre 2015, consulté le 30 avril 2019. URL : http://journals.openedition.org/rief/1074 ; DOI : 10.4000/rief.1074

Ce document a été généré automatiquement le 30 avril 2019.

\section{(c) (i) (9)}

Les contenus de la RIEF sont mis à disposition selon les termes de la Licence Creative Commons Attribution - Pas d'Utilisation Commerciale - Pas de Modification 4.0 International. 


\title{
Penser la langue, décrire le monde. Conversation avec Pierre Jourde
}

\author{
Veronic Algeri
}

v.A. Pierre Jourde, vous êtes critique littéraire et auteur de romans, mais aussi d'essais philosophiques et de théorie littéraire. Vous avez enseigné au collège, au lycée puis à l'université. Nombreux sont les professeurs qui sont aussi écrivains, mais vous, vous défendez un rapport à la littérature qui est total car de sa fonction sociale à ses lieux sacrés, de ses auteurs à ses destinataires, vous nous parlez de tout. Vous vous placez en tant que défenseur de son rôle et dénonciateur de la dérive de son système, face à une société qui semble ne plus s'y intéresser, et vous finissez par parler de ce monde qui n'est plus amoureux de son présent. Comment vivez-vous ce rapport total à la littérature?

P.J. «Rapport total» est une expression qui me convient assez. Elle signifie que la littérature est un engagement de soi, de sa vie. Je n'ai jamais pensé faire autre chose, j'ai organisé mon existence en fonction de la littérature, et si je suis devenu professeur, universitaire, c'est parce que c'était pour moi la meilleure façon de gagner ma vie en restant proche de la littérature. J'y vois aussi l'idée qu'un écrivain ne s'enferme pas nécessairement dans une pratique artistique limitée : il peut rechercher une sorte de totalité, en effet. Aborder, par la littérature, une grande variété de sujets, utiliser des genres divers. Peu d'écrivains français sont à la fois romanciers, poètes, essayistes, critiques, dramaturges, parodistes, etc. C'était plus souvent le cas autrefois. Je regrette que cette polyvalence se soit raréfiée.

Dans ce rapport, le risque est que la littérature finisse par l'emporter sur la vie. Pendant très longtemps, cela a été le cas pour moi. Rien n'était plus important. Cela a fini par changer. L'idéal serait que la littérature nous aide à naître au monde, qu'elle ne nous fasse pas lui tourner le dos, mais qu'elle soit une manière de rendre la vie plus vivante et plus intense. Ce n'est qu'un idéal.

v.A. Vous êtes un spécialiste de la littérature française que vous avez abordée à partir de différents points de vue : votre essai L'Alcool du silence sur la littérature fin de siècle analyse finement l'esprit de la décadence; dans votre ouvrage sur Vialatte vous rendez hommage à cet écrivain et à son esprit pascalien ; alors que dans Visages du double, que vous avez écrit avec Paolo Tortonese, c'est à un thème que vous consacrez votre analyse en traversant la 
littérature des mythes anciens au XXe siècle. Vous avez aussi travaillé sur la littérature contemporaine : en 1997 vous avez fondé avec, entre autres, Loïc Chotard, André Guyaux, Claudio Galderisi, Paolo Tortonese, la revue littéraire Hesperis, qui publie de jeunes auteurs, des traductions et des inédits, puis, encore, vous animez un blog dans lequel vous réagissez et réfléchissez à l'actualité. Quand vous parlez de la grande production littéraire qui caractérise le marché aujourd'hui, vous en dénoncez avec virulence les modes et la médiocrité : c'est le sujet de La Littérature sans estomac. Notre littérature, quand l'alerte de sa fin ne retentit pas dans la critique et dans les médias, ressemble désormais à un vaste ensemble sans écoles et sans modèles, où le marché et les prix littéraires nous pilotent vers quelques noms qui se vendent bien. Comment vous orientez-vous dans le panorama de la littérature contemporaine? Est-il encore possible de reconnaître des modèles littéraires, ou tout au moins des tendances?

P.J. La littérature n'a pas échappé au phénomène d'individualisation qui affecte les sociétés occidentales. Il n'y a plus de mouvements, il n'y a que des individus. Ou bien on se définit en fonction d'une appartenance ethno-sociologique, on est un écrivain femme, homosexuel, noir, breton, tunisien, à l'image d'une société fracturée en groupes défendant leurs particularités. Cette absence de groupes et de grandes tendances est à la fois une chance et un risque. La chance, c'est d'échapper au terrorisme intellectuel qui sévissait dans les années 1950-1960, à la soumission aux mots d'ordre et aux impératifs esthétiques, aux deux écueils du formalisme et du cloisonnement politique. Le risque, c'est l'absence de confrontation et de débat esthétiques, l'affirmation de la seule individualité, qui est en réalité une soumission aux lois du marché médiatique, qui exige de l'intime et de l'individu. Les tendances lourdes correspondent par conséquent à un retour à des modes de représentation réalistes, où dominent l'autofiction, le «biopic» ou le récit historique. Ce sont les livres primés et massivement lus. L'imagination a mauvaise presse, sauf dans des genres populaires comme l'heroic fantasy, qui peinent à se dégager de modes de représentation trop codifiés. Le grand succès de Houellebecq tient à ce qu'il est un des seuls à aborder de front les problèmes du monde réel, sous un angle à la fois réaliste et utopique, ou dystopique. La littérature française est aujourd'hui d'une très grande richesse, mais le problème consiste justement à faire connaître cette richesse, cette diversité, écrasée par les tendances lourdes.

v.A. Les adolescents, vous les connaissez bien de par votre travail d'enseignant et ils sont présents dans nombre de vos publications: Paradis noirs est un roman qui raconte la vie d'adolescents dans une institution religieuse; avec Carnets d'un voyageur zoulou, vous réagissez aux émeutes des banlieues de 2005; dans Festins secrets, vous dénoncez la culture jeune, sans repères et sans valeurs, qui pénètre notre société et le système scolaire entraînant la prééminence des valeurs d'épanouissement individuel et de loisirs sur celles de hiérarchie ou de devoir. Cette absence postmoderne de hiérarchie ressemble désormais au «présentisme » théorisé par François Hartog: une condition d'éternel présent, sans histoire et sans rêves. Parler des jeunes et de leur rapport à la culture est donc aussi une question de transmission du savoir. Comment envisagez-vous ce lien?

P.J. Le monde de l'enfance est en effet omniprésent dans mes textes, celui de l'adolescence également. Ce sont les âges métaphysiques, ceux auxquels on se confronte directement aux grandes questions. En tous cas ceux où on devrait normalement le faire. Je ne suis pas aussi complètement étranger à la "culture jeune ", que je connais tout de même pas mal, pour avoir élevé des enfants et fréquenté des adolescents. "Sans repères et sans valeurs " serait trop dire à mon sens. Ce que je dénonce en revanche, c'est le jeunisme comme produit industriel, c'est une culture factice, entièrement fabriquée par la démagogie médiatique. De sorte qu'on se retrouve avec certains jeunes 
qui ont, non plus des questions, ce qui serait normal, mais des réponses et des certitudes. Ça ne facilite pas la transmission. Mais je pense que la plupart des jeunes sont demandeurs d'une parole ferme, argumentée, pour autant qu'ils sentent de l'intérêt pour eux et de l'engagement dans ce qu'on dit. Le sens de l'humour est aussi essentiel dans cette relation. Je ne désespère pas, loin de là.

v.A. En 2003 paraît Pays perdu, un roman sur le petit hameau du Cantal dont vous êtes originaire, un hommage à cette population, à sa vie, épique en quelque sorte, et à ses valeurs naïves et vraies. Ce livre est très mal accueilli. Votre famille est agressée par les habitants du village qui sont décrits dans ces pages. Dix ans après, avec La première pierre, vous réagissez à cette violence de la littérature dont vous êtes accusé, et vous montrez que ce fameux clivage entre la paysannerie et l'intellectuel est une construction symbolique qui se forme à partir de stéréotypes. Alors que les acteurs de cet affrontement, la presse et les témoins, sont dans la fiction, vous en venez à comprendre que c'est à la littérature de reconstruire la vérité, pour ceux qui ont envie de la lire. Ce constat paradoxal est aussi le thème de votre ouvrage Littérature et authenticité, dans lequel vous abordez la relation de l'expérience à la littérature d'un point de vue philosophique. Face à ce grand malentendu, quelles sont aujourd'hui les valeurs perdues et, si elles ne sont pas dans nos pays perdus, où se trouvent-elles?

P.J. Je ne crois pas que le rôle de la littérature consiste à illustrer ou défendre des valeurs quelconques. Elle consiste bien plutôt à introduire un jeu dans les rouages des sens et des valeurs institués. Elle nous montre que nous ne sommes pas ce que nous sommes. Ce léger écart qu'elle introduit, on pourrait l'appeler, par exemple, liberté, résistance à la détermination. Tout ce qui fait qu'un paysan n'est pas qu'un paysan, par exemple. La littérature ouvre la possibilité de l'altérité et de la complexité. Sans doute est-elle elle-même soumise à des déterminations. Mais en introduisant de la complexité dans les mécanismes, elle rend cette détermination moins lourde, moins univoque. Il s'agit pour elle de défaire les fictions que nous nous sommes construites pour vivre, pour tenter de nous permettre une représentation un peu plus juste, un peu plus fine. Bien sûr nous sommes constitués de certains éléments donnés, fils de paysans, enfants d'immigrés, quels qu'ils soient. Mais ces éléments doivent cesser d'être les horizons indépassables de notre vérité pour devenir ce avec quoi nous allons construire quelque chose d'autre.

v.A. En même temps que vous dénoncez la crise des valeurs, un idéal moral fait surface et mène vos lecteurs à s'interroger sur l'ordre du monde. La ville de Logres, dans Festins secrets, est ce lieu où se condense le mal du monde, où le protagoniste accomplit une lente descente aux enfers pour connaître et révéler une horreur désormais banalisée qui s'inscrit au sein de notre quotidien. Dans ce roman, c'est une question d'espaces, insignifiants et indéterminés, et surtout une question de langues qui se posent: c'est le "Système " kafkaïen de l'éducation nationale, avec ses acronymes et son langage codé, et c'est «l'idiome lourd» des jeunes avec leur rap et leurs tags. Le niveau du signifiant est désormais incapable de coller au monde. Face à cette véritable maladie du langage que vous finissez par diagnostiquer, quel est dès lors le rôle de l'écrivain?

P.J. Festins secrets dénonce aussi la fascination du mal à l'œuvre dans le personnage principal, et sa propension à désespérer du monde. Le portrait qui y est dressé de notre monde doit être vu comme en partie vrai, en partie produit par la paranoïa et les fantasmes du narrateur. Mais il est vrai qu'il s'agit de montrer un signifiant en délire. Un processus de déréalisation à l'œuvre dans l'inflation des signes. L'écrivain ne fait pas confiance au langage. J'ai toujours pensé que la parole était "de trop », et le langage mensonger, ne serait-ce que parce qu'il est issu du désir de produire du langage, qui est aussi, plus ou moins, un désir de pouvoir et un désir d'être. Par conséquent, écrire, c'est 
commettre un acte intrinsèquement mensonger, et pourtant l'écriture détient la possibilité de sa rédemption, si elle parvient à un langage tel qu'il se défait pour ainsi dire du désir qui l'a suscité, s'il se neutralise. C'est une ascèse. Mais, alors, l'œuvre peut faire advenir ce qui nous fait essentiellement défaut: la grâce. Une manière d'être au monde telle que nous ne nous y imposons plus. Lire, c'est avoir la possibilité de connaître cette grâce, c'est s'en charger et être chargé de la transmettre.

v.A. Dégraissée, consistante, où le signe accompagne le sens dans cette révélation/ dénonciation, la langue est pour vous un véritable acte de langage. L'écriture devient un espace de tension à la fois littéraire et politique. Un langage qui pétrit l'espace, qui crée une image pourvue d'une force morale. L'approche sémiotique rejoint une approche philosophique, la fiction rejoint les faits réels... De quel côté se place la pulsion scopique du narrataire qui alimente les formes, les structures et les modes d'énonciation? Quels sont les modèles, s'il y en a dans ce sens, qui inspirent une telle approche?

P.J. Le narrataire est celui qu'il faut égarer dans la forêt. Il faut lui donner ce plaisir, ce trouble et ce frisson de l'égarement. Il y a ici et là des panneaux indicateurs, des «messages ", mais, comme dans un jeu de quête, on ne sait pas dans quelle mesure ils sont crédibles. Dans ce travail de déchiffrement, il doit, si du moins le dispositif fonctionne, mettre en jeu sa relation herméneutique au monde. Il a affaire à des personnages qui ne cessent de se construire des fictions. Il est donc invité à déconstruire les siennes, et c'est par là que, peut-être, la littérature est une propédeutique au réel.

v.A. Dans vos romans, la question du point de vue est centrale. Dans le récit à la deuxième personne, le narrataire s'adressant par un «tu » à son personnage fonde en quelque sorte son double: c'est le cas de ce petit bonhomme, dans La première pierre, comme de ce jeune professeur, dans Festins secrets. Ces personnages existent par le regard de l'autre, mais sont en même temps comme privés de leur vérité essentielle. Est-ce que ces personnages sont authentiques ou fabriqués? Ont-ils un statut d'objet ou de sujet? Pourquoi avez-vous recours à ce dispositif narratif?

P.J. Tous mes romans sont des discours. Pour certains, ce n'est pas évident au début, mais il apparaît petit à petit que quelqu'un s'adresse à quelqu'un. Dans La cantatrice avariée et dans L'heure et l'ombre, on ne comprend qu'à la fin qui parle à qui. Ce quelqu'un qui parle se situe parfois dans un espace difficilement situable par rapport au récit. Cela induit aussi des dispositifs d'énonciation étranges, ou complexes. Carnage de clowns est un récit à la première personne où le mot «je » ne figure pas. Le Maréchal absolu est constitué de quatre récits dans lesquels quatre narrateurs différents s'adressent à quatre narrataires à l'identité parfois conjecturale.

Il y a de multiples raisons à ces dispositifs d'énonciation. D'abord, je pense que l'intrigue, pour être bonne, doit atteindre les couches profondes du dispositif narratif. Par conséquent, l'énonciation devient un élément essentiel de l'intrigue. Cette intrigue porte le plus souvent sur une quête d'identité, passant par la parole, donc par la possibilité et le sens de dire « je », « tu », « il », « nous »... Dans Festins secrets, on ne sait pas vraiment qui dit «tu ». Est-ce le personnage principal qui s'adresse à lui-même ? Le narrateur qui s'adresse à lui ? Ou, bien plutôt, la voix intérieure d'un démon qui l'oriente vers le Mal ? Trouver sa parole, trouver sa place dans l'énonciation est la quête essentielle de mes personnages. Celui à qui l'on s'adresse ou celui qui parle détient souvent une sorte de puissance divine, ou maléfique. Ainsi la parole est aussi liée à l'éthique. Dans La première pierre, il faut que le moi qui parle dise «tu " parce que ce «je » qu'il se figurait détenir seul, comme instance de parole, il a découvert que son 
père pouvait aussi en faire usage. Il lui faut se dire « tu » pour sortir du « je » enfantin, et s'obliger à agir et à devenir. Le devenir est dans la parole.

v.A. En 2006 vous avez publié L'œuvre du propriétaire, parodie du travail universitaire appliqué à vos propres textes. Vous n'exemptez pas la critique littéraire de la parodie : vous êtes l'auteur, avec Eric Naulleau, d'un pastiche du Lagarde et Michard, Le Jourde et Naulleau, une analyse satirique des grands écrivains du XXe et XXIe siècle. Que pensez-vous de la provocation en littérature, est-ce désormais la dernière forme d'engagement digne d'être exercée ou le dernier des leurres que notre monde nous autorise? Quels sont les auteurs contemporains que vous dispensez de ce regard parodique?

P.J. Il ne s'agit pas de provocation. Dans notre esprit, trop de fausses valeurs, encensées par la critique, encombrent l'espace littéraire au détriment de vrais écrivains. D'autre part, on parle beaucoup autour des livres: on parle de l'auteur, on parle du sujet du livre, on parle des questions sociologiques ou historiques liées au livre, mais pas du texte. Le dispositif de la parodie de manuel scolaire permet de reproduire de larges extraits du texte, assortis de notes, d'exercices, de notices de présentation. Bref, même si cela est fait de manière plaisante, il s'agit de parler avant tout du texte, si négligé par les critiques. Lesquels s'y intéressent si peu qu'ils ont eu du mal à croire que les extraits authentiques que nous présentions n'étaient pas des parodies écrites par nous! Mais la critique continue à être mal perçue, mal acceptée, au pays du persiflage et de l'ironie. On peut dire tout ce qu'on veut des politiques, mais l'art et la littérature sont devenus sacrés, il ne faut pas y toucher. Cette sacralisation sent la mort.

Il y a une quantité d'auteurs contemporains passionnants aujourd'hui. Dans le grand chaos du discours sur la littérature, il importe de faire le tri. C'est pourquoi je défends Carole Martinez, Pierre Michon, Valère Novarina, Eric Chevillard, Marie-Hélène Lafon, Richard Millet, Marie Ndiaye, Frédéric Verger, Pierre Bergounioux, Marc Petit et quelques autres.

\section{INDEX}

Mots-clés : critique littéraire, individualisation, langage mensonger, dispositif narratif, quête d'identité 